\title{
Neural Coding of Sound Frequency by Cricket Auditory Receptors
}

\author{
Kazuo Imaizumi and Gerald S. Pollack \\ Department of Biology, McGill University, Montréal, Quebec H3A 1B1, Canada
}

Crickets provide a useful model to study neural processing of sound frequency. Sound frequency is one parameter that crickets use to discriminate between conspecific signals and sounds made by predators, yet little is known about how frequency is represented at the level of auditory receptors. In this paper, we study the physiological properties of auditory receptor fibers (ARFs) by making single-unit recordings in the cricket Teleogryllus oceanicus. Characteristic frequencies (CFs) of ARFs are distributed discontinuously throughout the range of frequencies that we investigated $(2-40 \mathrm{kHz})$ and appear to be clustered around three frequency ranges $(\leq 5.5,10-12$, and $\geq 18 \mathrm{kHz}$ ). A striking characteristic of cricket ARFs is the occurrence of additional sensitivity peaks at frequencies other than CFs. These additional sensitivity peaks allow crickets to detect sound over a wide frequency range, although the CFs of ARFs cover only the frequency bands mentioned above. To the best of our knowledge, this is the first example of the extension of an animal's hearing range through multiple sensitivity peaks of auditory receptors.

Key words: insect hearing; Teleogryllus oceanicus; frequency sensitivity; sound communication; ultrasound; acoustic startle response; phonotaxis
Sound frequency is an important carrier of information for many animals. The neural analysis of sound frequency begins with auditory receptor neurons. Understanding how the population of receptor neurons in an animal's ear represents sound frequency is required for a more general knowledge of the neural analysis of acoustic signals. One model system for studying the neurobiology of acoustic behaviors in general, and the analysis of sound frequency in particular, is the auditory system of crickets. In this paper, we describe the frequency sensitivity and selectivity of auditory receptors in the Australian field cricket Teleogryllus oceanicus.

Crickets hear sounds ranging in frequency from 2 to at least 100 $\mathrm{kHz}$ (Hoy et al., 1982), with $\sim 70$ auditory receptors in each ear (Young and Ball, 1974). Two narrower frequency regions within this wide range are of particular behavioral relevance. Sound energy in intraspecific signals is concentrated in relatively narrow spectral bands. In T. oceanicus, for example, intraspecific songs have dominant frequencies near $4.5 \mathrm{kHz}$ (Nolen and Hoy, 1986; Libersat et al., 1994; Balakrishnan and Pollack, 1996). The other class of behaviorally relevant sounds, the echolocation cries of aerially hunting bats, has energy in ultrasonic frequencies (Suga, 1990). Behavioral studies have shown that crickets have enhanced sensitivity to sounds in these two frequency ranges (Popov and Shuvalov, 1977; Moiseff et al., 1978; Pollack et al., 1984; Nolen and Hoy, 1986). Furthermore, crickets categorize the entire audible range into low frequencies and ultrasound, with a sharp boundary at $15 \mathrm{kHz}$ (Wyttenbach et al., 1996).

In the past, only a few studies have addressed the physiology of cricket auditory receptors (Zaretsky and Eibl 1978; Esch et al., 1980; Hutchings and Lewis, 1981; Oldfield et al., 1986). Most of

Received Sept. 15, 1998; revised Nov. 30, 1998; accepted Dec. 3, 1998.

This work was supported by grants from the Natural Sciences and Engineering Research Council of Canada and the Whitehall Foundation to G.S.P. and in part by a Government of Canada award to K.I.

Correspondence should be addressed to Gerald S. Pollack, Department of Biology, McGill University, 1205 Avenue Dr. Penfield, Montréal, Quebec H3A 1B1, Canada.

Copyright (ㄷ) 1999 Society for Neuroscience $\quad 0270-6474 / 99 / 191508-09 \$ 05.00 / 0$ these failed to investigate sensitivity to ultrasound, either because of technical reasons or because they were performed before the behavioral significance of ultrasound was appreciated. Moreover, these studies described responses at threshold sound intensities and thus neglected the suprathreshold intensities at which most acoustic behaviors occur (Capranica, 1992).

In this paper, we address the general physiological properties of cricket auditory receptors. Our main goal is to explore sensitivity to different frequencies at suprathreshold intensities and frequency selectivity at threshold, as well as suprathreshold intensities.

Part of the work has been published previously in preliminary form (Imaizumi and Pollack, 1996, 1997).

\section{MATERIALS AND METHODS}

Animals. Australian field crickets, T. oceanicus, were cultured on a $12 \mathrm{hr}$ dark/light cycle at $25-28^{\circ} \mathrm{C}$ in our laboratory colony. They were kept in a crowded population and fed water and cat chow (Ralston Purina, St. Louis, MO) ad libitum. Only adult virgin females, 14-23 d after the final molt, were used.

Recording procedures. Animals were waxed on a platform ventral side up after removal of the wings, midlegs, and hindlegs. The femur of each front leg was fixed, with warm wax, perpendicular to the cricket's longitudinal axis. The tibia was held flexed against the femur. The prothoracic ganglion, which is where auditory receptors terminate (Eibl and Huber, 1979), was exposed by ventral dissection and kept moist with modified TES ringer (Strausfeld et al., 1983; Pollack, 1994). The ganglion was stabilized by a silver platform, and a chlorided silver ground wire was placed into the abdomen. Experiments were performed in a soundattenuating chamber at $20-23^{\circ} \mathrm{C}$.

We used two different recording techniques: whole tympanal nerve (WTN) and single-unit recordings. The WTN recordings monitored compound action potentials of a population of auditory receptor fibers (ARFs). Two Teflon-insulated silver wires (outer diameter, $114 \mu \mathrm{m}$ ), the tips of which were bare, were inserted into the femur close to the nerve that carries the axons of ARFs. To eliminate efferent activity from the recordings, the leg nerve was cut between the coxa and prothoracic ganglion. Nevertheless, spontaneous activity was not eliminated (see Fig. 4). Single-unit recordings were made with glass micropipettes, filled with either $3 \mathrm{M} \mathrm{KCl}$ (resistance, $10-40 \mathrm{M} \Omega$ ) or $2-4 \%$ Lucifer yellow $\mathrm{CH}$ (Aldrich, Milwaukee, WI) in distilled water (resistance, 100-300 M 2 ) in the leg nerve near its entry into the prothoracic ganglion. The anatomy of axon terminals of ARFs as revealed by injection of Lucifer yellow is described elsewhere (Imaizumi and Pollack, 1996, 1998). 
R96030101

R97030607
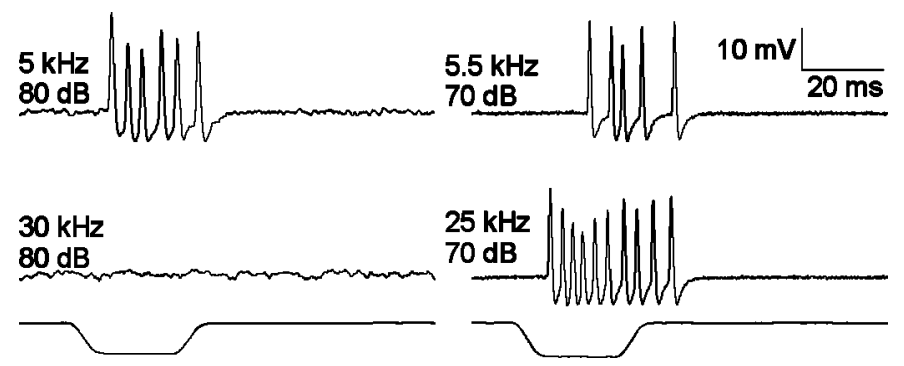

Figure 1. Single-unit recordings from two different ARFs. ARFs respond in a tonic manner. Stimulus frequency and intensity are given at the left of the traces. Stimulus monitors are shown below the traces. Calibration applies to all traces.

Acoustic stimulation. Sound stimuli were generated by a National Instruments (Austin, TX) AT-MIO-64F5 input-output board (resolution, 12 bits; digital-to-analog update rate, $250 \mathrm{kHz}$ ) driven by software written using LabWindows/CVI (National Instruments). After power amplification (Amcron) and computer-controlled attenuation (Mike Walsh Electronics), stimuli were delivered either through 4-inch magnetic speakers (InterTAN, Fort Worth, TX) for lower frequencies $(\leq 10$ $\mathrm{kHz}$ ) or through piezoelectric speakers (Matsushita Electric Industrial) for higher frequencies $(>10 \mathrm{kHz})$, ipsilateral to the recording side. The fundamental frequency was more intense than all harmonics by at least 45-50 dB. Increments between sound frequencies were $0.5 \mathrm{kHz}$ for stimuli in the range of $2-6 \mathrm{kHz}, 1 \mathrm{kHz}$ in the range of $6-10 \mathrm{kHz}, 2 \mathrm{kHz}$ in the range of $10-20 \mathrm{kHz}$, and $5 \mathrm{kHz}$ in the range of $20-40 \mathrm{kHz}$. Sound intensity (re. $20 \mu \mathrm{Pa}$ ) was calibrated with a 1/4-inch Brüel \& Kjaer 4135 condenser microphone and 2610 measuring amplifier. Sound stimuli were $30 \mathrm{msec}$ in duration (including $5 \mathrm{msec}$ rise and fall times) and were presented at two pulses per second. The search stimulus used was either $4.5 \mathrm{kHz}$ (or $5 \mathrm{kHz}$ ) at $80 \mathrm{~dB}$ sound pressure level (SPL) or bandpass filtered white noise $(3-50 \mathrm{kHz})$ at $90 \mathrm{~dB}$ SPL.

Data analysis. Responses were stored on magnetic tape and were digitized (sampling rate, $10 \mathrm{kHz} / \mathrm{channel}$ ) and analyzed using the computer program SWEEPS (Pollack, 1997). The WTN recordings were bandpass filtered (100-1000 Hz), full-wave rectified, and integrated over a $40 \mathrm{msec}$ time window beginning at stimulus onset. For single-unit recordings, the number of spikes was counted in a $50 \mathrm{msec}$ time window beginning at stimulus onset, except for one ARF with unusually long latency in which the window began $10 \mathrm{msec}$ after stimulus onset.

We describe ARFs according to a number of parameters. Characteristic frequency (CF) is normally defined as the frequency at which threshold is the lowest. However, because cricket auditory receptors have extremely small axons, we were not always able to maintain recordings long enough to measure threshold. In these cases, we took as CF the best frequency $(\mathrm{BF})$ that, at the lowest suprathreshold intensity tested, elicited the largest number of spikes. BF is usually defined as the frequency that elicits the largest number of spikes at a given sound intensity. We used this definition in most cases, but we departed from it for some ultrasound ARFs. As shown in Figure $3 E$, some ultrasound ARFs are sensitive to 10 and/or $16 \mathrm{kHz}$, as well as to ultrasound. At high sound intensity, they may produce a larger number of spikes to 10 or $16 \mathrm{kHz}$ than to ultrasound, although a peak in spike count near the CF is still evident. Strictly speaking, the BF at these intensities would be 10 or 16 $\mathrm{kHz}$. However, to ensure that single ARFs maintained a single identity through the analysis, we took as BF the frequency of the response peak closest to CF. Spontaneous rate was determined from recordings 2-20 sec (primarily $6-15 \mathrm{sec}$ ) in duration without sound stimulation. Threshold intensity was estimated from nonlinear curves fit to intensity-response data (Yates, 1990) as the sound intensity at CF at which an ARF produced one spike above the spontaneous rate. Curves were fit using Sigma Plot (SPSS, Chicago, IL). In most cases, a five-parameter sigmoid model was used. For ARFs with high threshold, either a four-parameter sigmoid or logistic model was applied.

\section{RESULTS}

We made single-unit recordings from more than 120 ARFs. ARFs respond to sound in a tonic manner (Fig. 1). Because the axons of cricket ARFs are too small to make prolonged stable
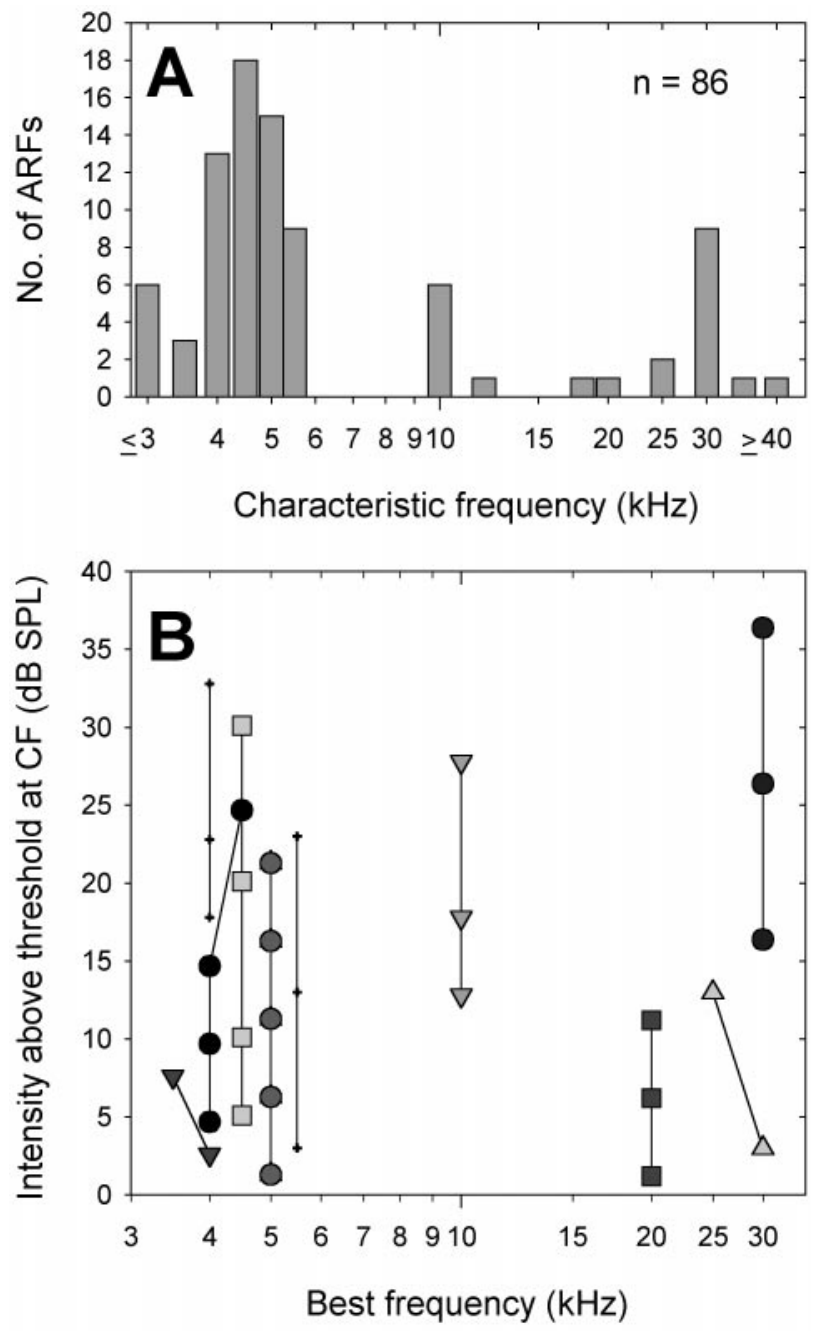

Figure 2. A, The distribution of CFs of 86 ARFs. CFs of $3 \mathrm{kHz}$ and lower and $40 \mathrm{kHz}$ and higher are expressed as $\leq 3$ and $\geq 40$, respectively. ARFs appear to fall into three populations based on $\mathrm{CF}(\leq 5.5,10-12$, and $\geq 18$ $\mathrm{kHz}$ ). $B$, The relationship between the shift of best frequency and stimulus intensity. Ten representative ARFs are illustrated by different symbols.

single-unit recordings, the mean recording duration was $\sim 3$ min (range of 1-11 $\mathrm{min}$ ).

\section{Distribution of CF}

We determined the CFs of 86 ARFs. They appear to fall into three populations based on their CF: low-frequency $(\leq 5.5 \mathrm{kHz})$, mid-frequency $(10-12 \mathrm{kHz})$, and ultrasound ( $\geq 18 \mathrm{kHz})$ ARFs (Fig. $2 A)$. Three-quarters $(74.5 \%)$ of ARFs had CFs from $\leq 3$ to $5.5 \mathrm{kHz}$. Among these, a majority showed CFs from 4 to $5.5 \mathrm{kHz}$. The remaining CFs were $10-12 \mathrm{kHz}(8 \%)$ or in the ultrasound range, $\geq 18 \mathrm{kHz}(17.5 \%$ ) (Fig. $2 A)$. We did not encounter any ARFs with CFs in the range of $6-9 \mathrm{kHz}$. Seven ARFs appeared to have CFs outside of the range we investigated $(3-40 \mathrm{kHz}$ in the early experiments, $2-40 \mathrm{kHz}$ in the later ones).

We determined $\mathrm{CF}$ from $\mathrm{BF}$ at the lowest suprathreshold intensity tested (see Materials and Methods). If the BF changes with stimulus intensity, our determination may not be appropriate. In vertebrate auditory nerve fibers (ANFs), for instance, BF is shifted to lower frequencies with increasing stimulus intensities (Møller, 1977; Capranica, 1992; Sams-Dodd and Capranica, 1994). Figure $2 B$ illustrates that BF was fairly stable in those 

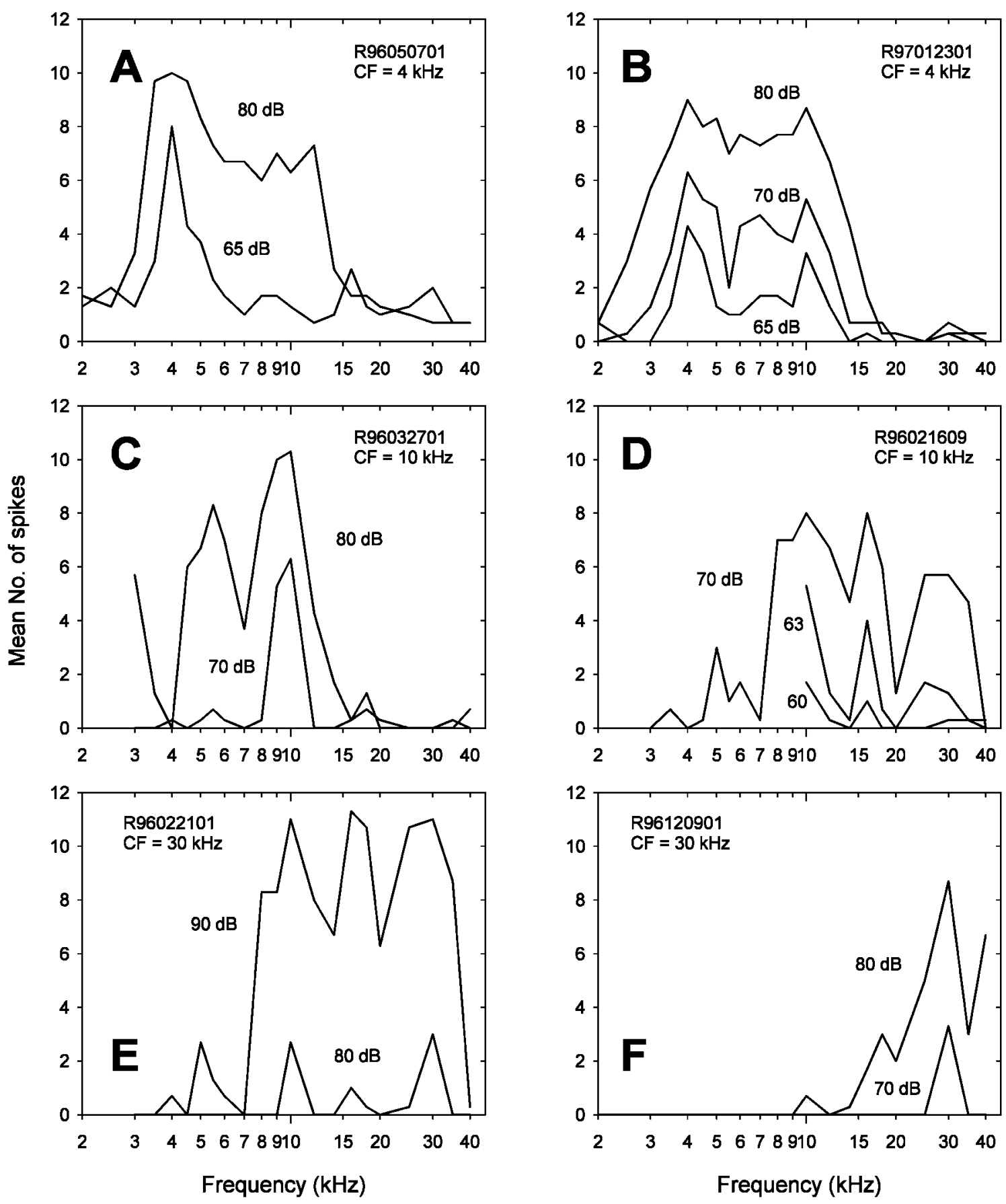

Figure 3. Iso-intensity curves of six representative ARFs. The mean number of spikes from three responses is illustrated as a function of frequency. ARF identifications and their CFs are shown on the top left or right. Stimulus intensity is given beside each iso-intensity curve. $A$ and $B$ are low-frequency ARFs. $C$ and $D$ are mid-frequency low- and high-pass ARFs, respectively. $E$ and $F$ are ultrasound ARFs. See Results for further explanation.

ARFs that we tested over a wide range of intensities. The changes that did occur were all to neighboring frequencies that we examined (see Materials and Methods for ultrasound ARFs) (Fig. 2B). Therefore, our determination of $\mathrm{CF}$ from BF at the lowest suprathreshold intensity tested is appropriate.

\section{Iso-intensity responses of ARFs}

Most low-frequency ARFs are similar to one another in their patterns of frequency sensitivity. They are fairly selective at low intensities, and at higher intensities, they exhibit additional sensitivity peaks to higher sound frequencies, generally in the range of 8-12 kHz (Fig. 3A). A small number of low-frequency ARFs had another sensitivity peak even at low intensities (Fig. $3 B$ ). We found two types of mid-frequency ARFs based on their frequency filtering properties. One of these, which we refer to as midfrequency low-pass ARFs, responds well to CF and lower (Fig. $3 C)$. The other type, mid-frequency high-pass ARFs, responds to CF (10 or $12 \mathrm{kHz}$ ) and higher (Fig. 3D). We also found two types of ultrasound ARFs. One type is almost as sensitive to 10 or 16 $\mathrm{kHz}$ as to ultrasound (Fig. $3 E$ ). The other type is sensitive only to ultrasound at low intensities (Fig. $3 F$ ). 


\section{WTN96080101}
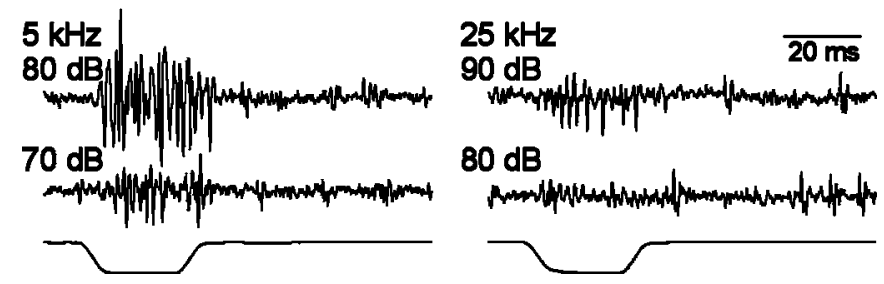

Figure 4. Extracellular recordings from the WTN in one animal. Stimulus frequency and intensity are given at the left of the traces. Stimulus traces are shown at bottom. Scale bar applies to all traces.

\section{Our sample of ARFs is representative of the entire population of auditory receptors}

It is extremely unlikely, given the sample size of our single-unit recordings $(n=86)$, that we have recorded from each of the $\sim 70$ receptors in the cricket's ear. To determine whether our sample is representative of the entire receptor population, we compared WTN recordings with single-unit recordings. Examples of WTN recordings are shown in Figure 4. These recordings reflect the summed activity of all ARFs that respond to the stimulus. The conspicuous differences in amplitude of compound action potentials elicited by low frequency and ultrasound are attributable to the differences of the number of receptors responding to these frequencies (Pollack and Faulkes, 1998). Figure $5 A$ summarizes WTN response amplitude from 10 different ears in response to 80 $\mathrm{dB}$ stimuli. Figure $5 B$ shows the total number of spikes produced by 80 ARFs, recorded as single units, to the same stimuli (we excluded six ARFs for which we did not have responses at $80 \mathrm{~dB}$ over the entire frequency range). The iso-intensity curve for the summed single-unit responses is quite similar to the WTN recording, with the exception that the small peak visible at $30 \mathrm{kHz}$ in the summed single-unit responses is not evident in the WTN recordings (Fig. $5 A, B$ ). The overall similarity of the two curves, however, suggests that our sample of ARFs is reasonably representative of the entire population of auditory receptors. Nevertheless, we cannot exclude the possibility that we have missed some receptor types.

\section{Additional sensitivity peaks}

The iso-intensity curves of Figure 3 show that single ARFs may exhibit sensitivity peaks at several frequencies in addition to their CF. To examine this phenomenon more systematically, we adopted a quantitative criterion for the identification of additional peaks. Sensitivity peaks were identified at those frequencies that elicited spike counts above the threshold level (see Materials and Methods) and were flanked by troughs in which the response dropped to $75 \%$ of that at the peak. Sensitivity peaks occasionally spanned two to three frequencies. In these cases, if mean spike count was greatest at one of these frequencies, this was taken as the position of the sensitivity peak. If spike counts were equal, the position of the peak was taken either as the frequency at which the SD of the response of the ARF was the lowest or as the mean of the frequencies, if the SDs were the same. Figure 6 illustrates additional sensitivity peaks of two ARFs. There are two sensitivity peaks in Figure $6 A(a$ and $b)$ and three in Figure $6 B(c-e)$. Sensitivity peaks $a$ and $c$ occurred at BF (see Materials and Methods), and $b, d$, and $e$ were additional sensitivity peaks. In general, the frequencies of additional sensitivity peaks remain stable with increasing intensity. In Figure $3 D$, for example, an additional sensitivity peak is apparent at $16 \mathrm{kHz}$ for all intensities
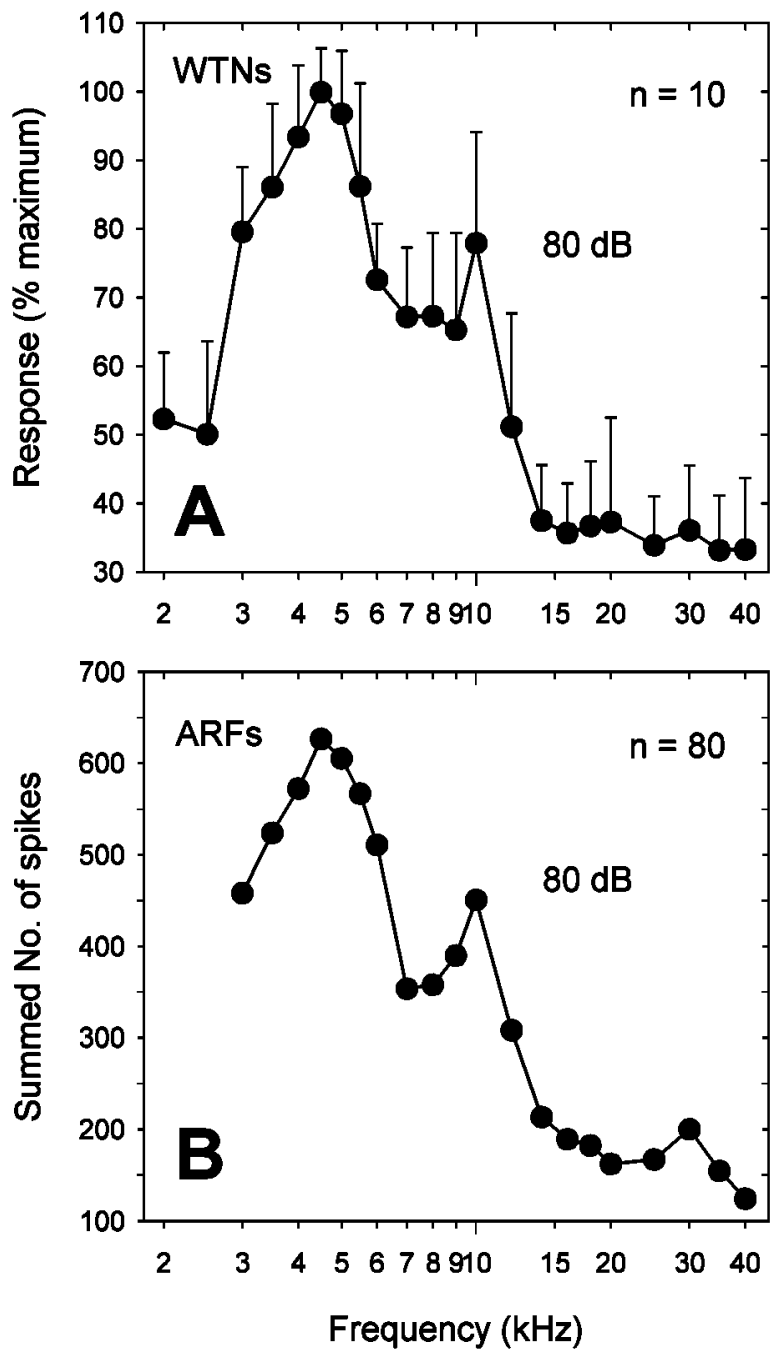

Figure 5. Comparison between WTN and single-unit recordings. $A$, WTN recordings were filtered, full-wave rectified, integrated, and normalized to the maximum response. Responses from 10 different ears were averaged and normalized again. In each WTN recording, 3-10 repetitions were presented at each frequency. Error bars indicate SDs. $B$, Summed spike counts of 80 ARFs stimulated over the range of $3-40 \mathrm{kHz}$ at $80 \mathrm{~dB}$. See Results for further explanation.

illustrated. Rarely ( $\sim 7 \%$ of additional sensitivity peaks), the additional sensitivity peaks shifted to neighboring stimulus frequencies as intensity increased. In these cases, we designated additional sensitivity peaks according to their frequencies at the lowest sound intensities at which they appeared. The number of additional sensitivity peaks in single ARFs varied from one to five.

In a sample of $70 \mathrm{ARFs}$ (most of them tested at more than one intensity), 132 additional sensitivity peaks occurred. In Figure 7, the positions of additional sensitivity peaks are plotted as a function of $\mathrm{CF}$. The diameter of each point corresponds to the sample size. The points above and below the diagonal dashed line represent additional sensitivity peaks at frequencies above and below CF, respectively. Although we did not find any ARFs with CFs of $6-9 \mathrm{kHz}$, nor with CFs between $12-18 \mathrm{kHz}$, additional sensitivity peaks in these ranges are not uncommon. Interestingly, most ARFs, including mid-frequency high-pass and ultrasound ARFs, show sensitivity peaks to $4-5.5 \mathrm{kHz}$ at high intensities (e.g., Figs. 1, 3D,E). This suggests that most ARFs may respond to intraspecific signals at high intensity. 

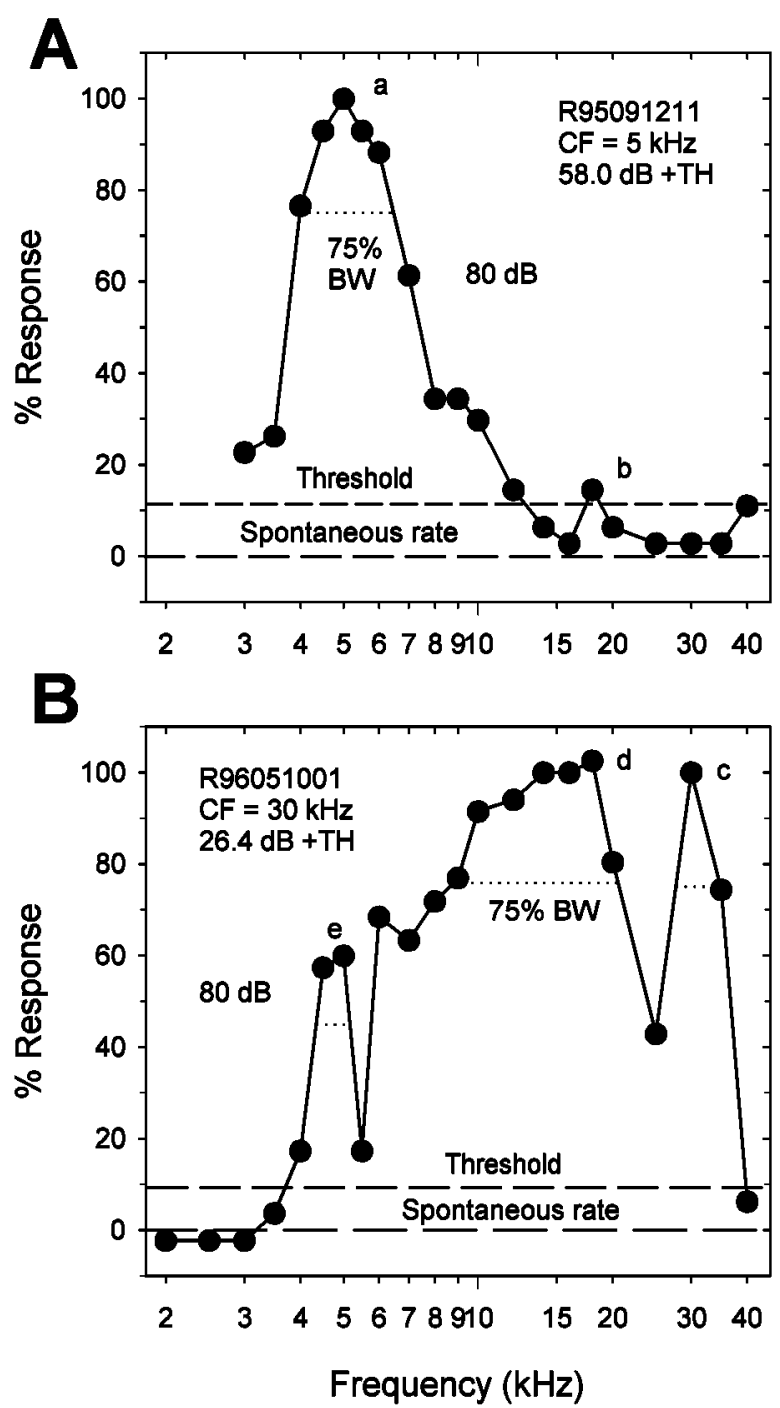

Figure 6. Criteria for sensitivity peaks. Iso-intensity responses were normalized to the number of spikes at BF (see Materials and Methods) after spontaneous rate was subtracted. Sensitivity peaks occurred at frequencies that elicited responses above threshold levels (see Materials and Methods) and were flanked by troughs in which the responses dropped to $75 \%$ of the peak. Peaks in $A(a)$ and $B(c)$ occurred at BF. Additional sensitivity peaks occurred at $A(b)$ and $B(d, e)$. Spontaneous rates, threshold, and $75 \%$ bandwidth $(75 \% \mathrm{BW})$ are illustrated by different dashed lines. ARF identifications, $\mathrm{CFs}$, and sound intensity above threshold $(+T H)$ are given at the top left or right. Both $A$ and $B$ are iso-intensity curves at $80 \mathrm{~dB}$. $A$, Low-frequency ARF with unusually low threshold and long latency. This ARF maintained its frequency selectivity, even at nearly $60 \mathrm{~dB}$ above threshold. $B$, Ultrasound ARF. This ARF was almost equally sensitive to 16 and $30 \mathrm{kHz}$ at threshold. Additional sensitivity peaks occurred at 5 and $18 \mathrm{kHz}$.

The occurrence of multiple sensitivity peaks in single ARFs should serve to extend the cricket's audible range beyond that covered by the CFs of their ARFs. In Figure 8, we plot the $75 \%$ bandwidths of ARFs (Fig. 6) at 70 and $80 \mathrm{~dB}$. Each row in Figure 8 represents a single ARF. We could not determine bandwidths for ARFs with CFs near the ends of the range of frequencies tested, because their response curves were truncated on one side. Thus, we include only ARFs with CFs of $4-35 \mathrm{kHz}$ in Figure 8. At $70 \mathrm{~dB}$, the population of bandwidths leaves a gap in the frequency range from 6 to $10 \mathrm{kHz}$, but at $80 \mathrm{~dB}$, the entire frequency range is filled in by the bandwidths of ARFs (Fig. 8). These data show that crickets can hear a wide range of sound frequencies, even though they lack ARFs tuned to some portions of the range.

Additional sensitivity peaks are not distributed randomly across the range of audible frequencies. Two factors may contribute to their distribution. Figure $9 A$ shows that additional sensitivity peaks are clustered at $\sim 4-6$ and $8-12 \mathrm{kHz}$. There are hints of additional clusters near $15 \mathrm{kHz}$ and from $25-35 \mathrm{kHz}$, as well. Thus, additional sensitivity peaks occur near the dominant frequency of intraspecific signals and its higher harmonics.

In Figure $9 B$, the positions of additional sensitivity peaks are plotted as multiples of the CFs of the ARFs in which they occur. We excluded ARFs with CFs $\leq 3$ or $\geq 40 \mathrm{kHz}$. Here, it is evident that the additional sensitivity peaks tend to occur at subharmonics and higher harmonics of the CF. This suggests that the positions of the peaks may be set by factors (e.g., mechanical resonance) similar to those that determine the value of the $\mathrm{CF}$.

The cluster of additional sensitivity peaks at $4-6 \mathrm{kHz}$ is derived from all ARF populations (Fig. 9A). Approximately half of the low-frequency ARFs contributing to this cluster had CFs of 3 $\mathrm{kHz}$ or lower (e.g., Fig. 7). Additional sensitivity peaks near 10 $\mathrm{kHz}$ occurred primarily in low-frequency ARFs with CFs of $4.5-5.5 \mathrm{kHz}$ and to a lesser extent in ultrasound ARFs. Additional sensitivity peaks near $16 \mathrm{kHz}$ occurred in all populations. Additional sensitivity peaks at $20-35 \mathrm{kHz}$ were primarily derived from low-frequency ARFs (Figs. 7, 9A). However, most of these peaks were not consistent and disappeared at higher intensities (e.g., Fig. 3A).

In Figure $9 B$, the most conspicuous clusters of additional sensitivity peaks occurred at $1 / 6,1 / 3,1 / 2$, and 2 times the CF. The clusters at the subharmonics derive primarily from $10 \mathrm{kHz}$ and ultrasound ARFs, and those at higher harmonics are primarily from low-frequency ARFs.

\section{Frequency selectivity at $\mathrm{CF}$}

We quantified frequency selectivity by computing the quality factor at $75 \%\left(Q_{75 \%}\right)$, defined as BF divided by the bandwidth at $75 \%$ of the normalized response at a given intensity (Fig. 6). The larger the $Q_{75 \%}$ value, the greater the frequency selectivity. To compare frequency selectivity among the ARF populations, we chose two narrow intensity ranges, from 0 to $<5 \mathrm{~dB}$ above threshold (Fig. 10 $A$ ) and from 10 to $<15 \mathrm{~dB}$ above threshold (Fig. $10 B) . Q_{75 \%}$ varies widely among ARFs. At near-threshold intensity, there appears to be a tendency for low-frequency ARFs to be more sharply tuned than ultrasound ARFs, although the difference is not significant (Fig. 10A). At higher intensity, the $Q_{75 \%}$ values of the three ARF populations are similar (Fig. 10B).

ARFs become less sharply tuned as intensity increases. The relationship between $Q_{75 \%}$ and stimulus intensity is shown in Figure $11 A$ for a few representative ARFs. $Q_{75 \%}$ decreases with increasing stimulus intensity. Regression lines for a larger number of ARFs are shown in Figure $11 B$.

\section{DISCUSSION}

Our results demonstrate that the wide frequency range of cricket hearing is mediated by a discontinuous population of receptor neurons, the CFs of which are clustered around a few frequency regions within the audible range. The overall hearing range extends beyond that covered by the CFs of ARFs, because ARFs exhibit distinct sensitivity peaks at frequencies other than the CF. This situation differs from that found in the ears of higher vertebrates and bushcrickets, which also have extended hearing ranges. 


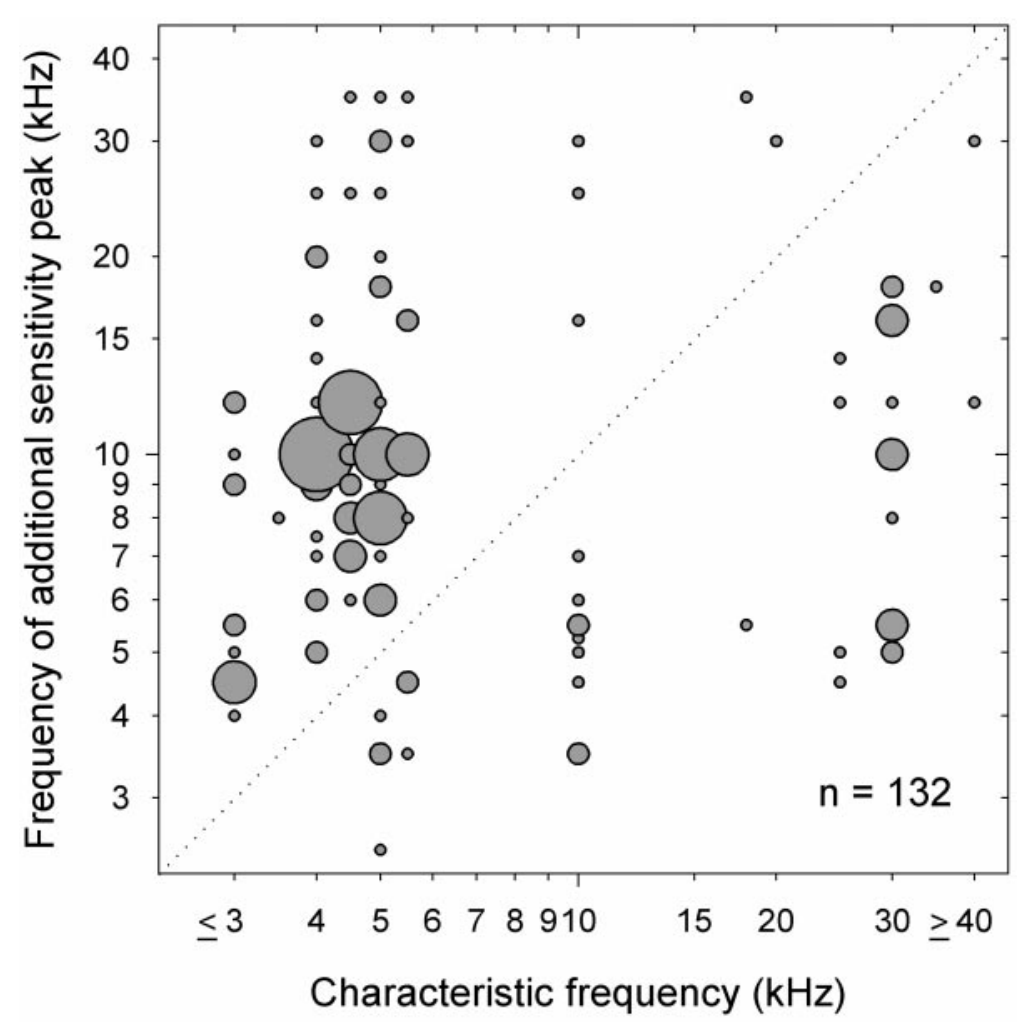

Figure 7. Frequencies of 132 additional sensitivity peaks are plotted as a function of CF. The diameter of each point is proportional to the number of additional sensitivity peaks occurring at those coordinates (the smallest points correspond to $n=$ 1). The diagonal dashed line separates additional sensitivity peaks occurring at frequencies above and below CF.
In these animals, the broad range of hearing is matched by a similarly broad range of CFs of receptor neurons (cats: Kiang, 1965; bushcrickets: Römer, 1983; Stumpner, 1996). Our results are, to our knowledge, the first to demonstrate that a broad range of hearing is generated by multiple sensitivity peaks of receptor neurons.

\section{Distributions of CFs}

Frequency sensitivity of cricket ARFs has been reported in three papers (Zaretsky and Eibl, 1978; Esch et al., 1980; Hutchings and Lewis, 1981). In all three cases, as in the present study, the largest population of ARFs had CFs near the dominant frequency of the calling song. Two of the studies (Zaretsky and Eibl, 1978; Esch et al., 1980) were limited to frequencies $\leq 20 \mathrm{kHz}$, and thus ultrasound ARFs were unlikely to have been detected. In contrast, Hutchings and Lewis (1981) used frequencies up to $42 \mathrm{kHz}$ and, like us, studied T. oceanicus. However, they did not describe CFs in sufficient detail for a direct comparison with our results.

Discontinuous distributions of CFs are often found in lower vertebrate ANFs (frogs: Feng et al., 1975; Narins and Capranica, 1976; lizards: Weiss et al., 1976), and, as for crickets (see below), reflect the frequencies of behaviorally relevant sounds (Simmons and Buxbaum, 1996).

\section{Frequency selectivity of ARFs}

Frequency selectivity, measured as the $Q_{75 \%}$ bandwidth of the sensitivity peak corresponding to the BF, was not significantly different among the different populations of ARFs. However, there appears to be a trend toward greater selectivity, near threshold, of low-frequency ARFs (Fig. 10A).

In vertebrate ANFs, frequency selectivity tends to increase with CF (Kiang, 1965; Liberman, 1978; Sachs et al., 1980; Eatock et al., 1981; Salvi et al., 1992; Sams-Dodd and Capranica, 1994; Köppl 1997). In cricket ARFs, a similar tendency is absent.

\section{Multiple sensitivity peaks}

A striking characteristic of cricket ARFs is the occurrence of multiple sensitivity peaks. This property was reported previously for crickets by Esch et al. (1980) and Hutchings and Lewis (1981), who found $\sim 10 \%$ of the ARFs they recorded showed two distinct frequency optima. Hutchings and Lewis (1981) also illustrated many ARFs that appeared to have several distinct frequency optima (e.g., their Figs. 5, 6). In our experiments, which included suprathreshold intensities, multiple sensitivity peaks occurred in most ARFs. Multiple sensitivity peaks are not unique to cricket ARFs. They have also been observed in locusts (Michelsen, 1971a; Inglis and Oldfield, 1988). The multiple sensitivity peaks of locust ARFs could be explained by the relationship between the spatially distributed resonances of the tympanal membrane and the locations of receptors (Michelsen, 1971a,b).

It seems likely that, in crickets too, multiple sensitivity peaks may have their origin in the still unknown biophysical mechanisms underlying the frequency selectivity of ARFs. The main sound-collecting structure, the posterior tympanal membrane, is broadly tuned to the dominant frequency component of the species calling song (Paton et al., 1977; Larsen, 1987; but see Johnstone et al., 1970). However, its tuning is not sharp enough to account for the selectivity of ARFs with CFs near this frequency. Furthermore, the decreasing sharpness of tuning with increasing intensity (Fig. 11) is not accounted for by the linear response of the tympanal membranes to increasing intensity (Paton et al., 1977). It seems clear that there are one or more additional stages of filtering (Kalmring et al., 1978), which might reside in the mechanics of the inner ear (Kalmring and Jatho, 1994) and/or within the receptor neurons themselves (Oldfield, 1985). A large proportion of additional sensitivity peaks occurs at frequencies of 4-6 kHz (Fig. 9A), i.e., near the dominant frequency of communication signals in this species. This may be attributable in part to 
Figure 8. Representation of a wide range of audible frequencies by ARFs. Each row represents $75 \%$ bandwidth(s) of sensitivity peak(s) from a single ARF. The plots for 80 and $70 \mathrm{~dB}$ show the bandwidths of 50 and 40 ARFs, respectively. Only ARFs with CFs of $4-35 \mathrm{kHz}$ and that continued to exhibit a clear sensitivity peak at BF (as defined in Materials and Methods) at these relatively high intensities were included.

the inability of additional stages of filtering to overcome the relatively large vibration of the tympanum in this frequency range (Johnstone et al., 1970; Paton et al., 1977). The tendency toward a harmonic relationship between frequencies of additional sensitivity peaks and the $\mathrm{CF}$, evident in Figure $9 B$, also points to additional stages of filtering as the most likely site for generation of these peaks.

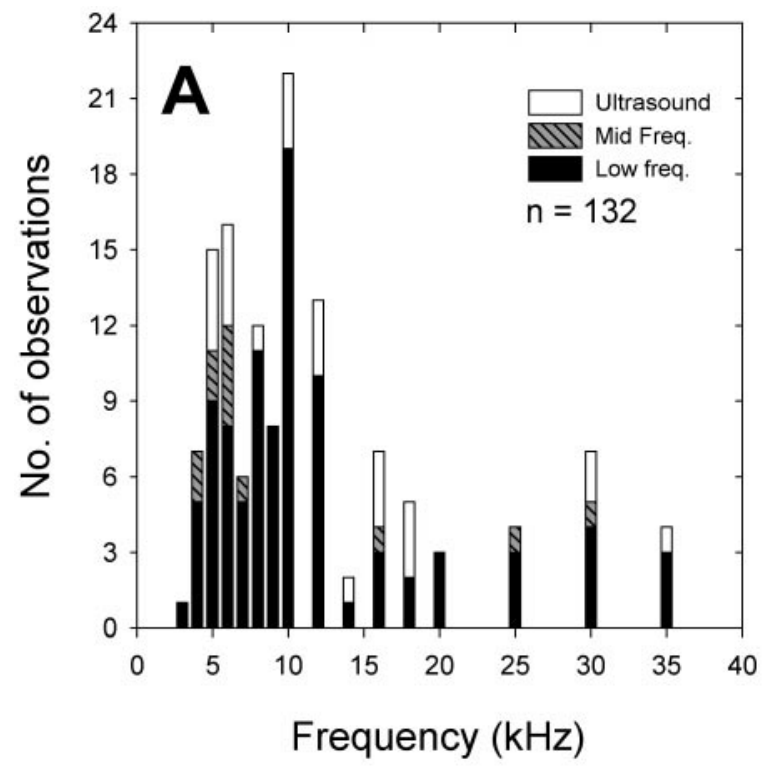

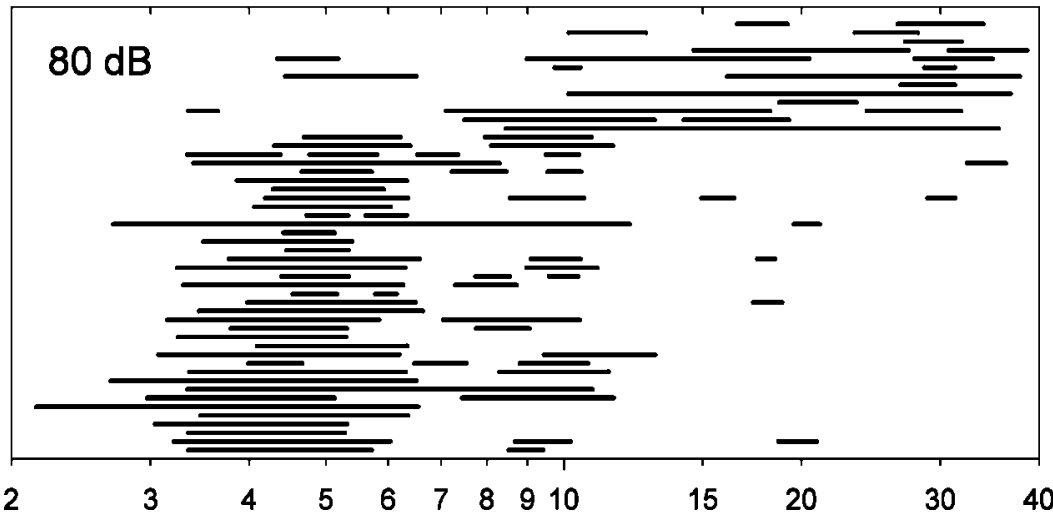

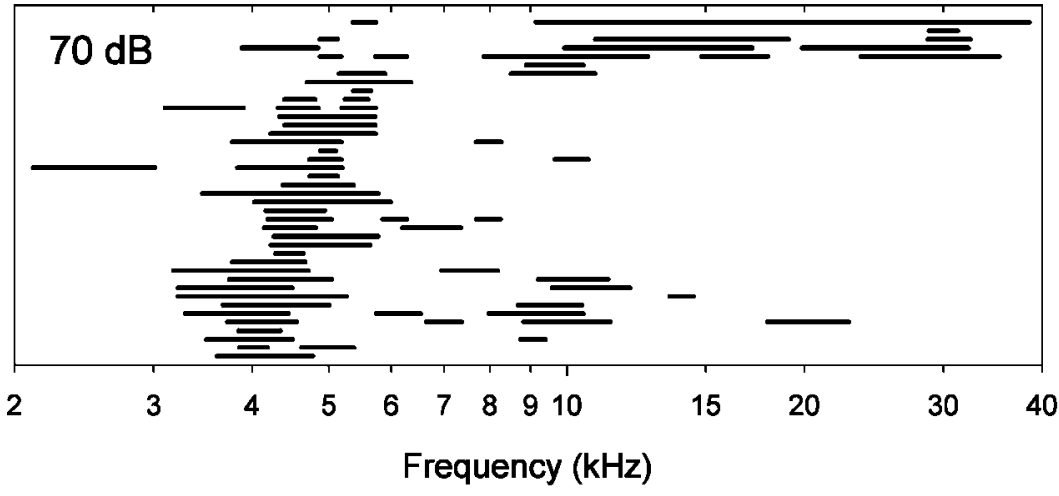

Behavioral roles of the different ARF populations

Two of the three ARF populations, the low-frequency and ultrasound ARFs, seem clearly related to behavior. T. oceanicus communicates using acoustic signals with dominant frequencies near $4.5 \mathrm{kHz}$, i.e., in the same range as the CFs of the majority of low-frequency ARFs (Fig. 2A). Ultrasound stimuli elicit startle or avoidance responses, which may offer protection against preda-

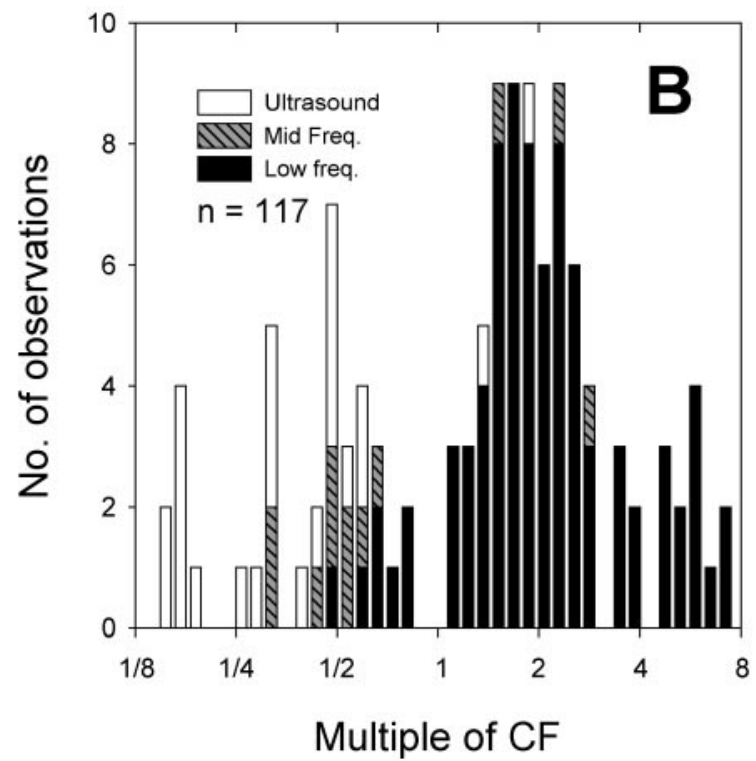

Figure 9. Distributions of additional sensitivity peaks. A, Additional sensitivity peaks are plotted according to the frequencies at which they occur. The origins of ARF populations contributing the additional sensitivity peaks are illustrated with different fill patterns. Peaks are clustered at the frequency of intraspecific signals $(4-6 \mathrm{kHz})$ and its higher harmonics. $B$, Additional sensitivity peaks are plotted as multiples of the CF of the ARF in which they occur. For this analysis, ARFs with CFs $\leq 3$ or $\geq 40 \mathrm{kHz}$ were excluded. Peaks are clustered at harmonics of the CF (1/6, 1/3, 1/2, and 2 times the CF). See Results for further explanation. 

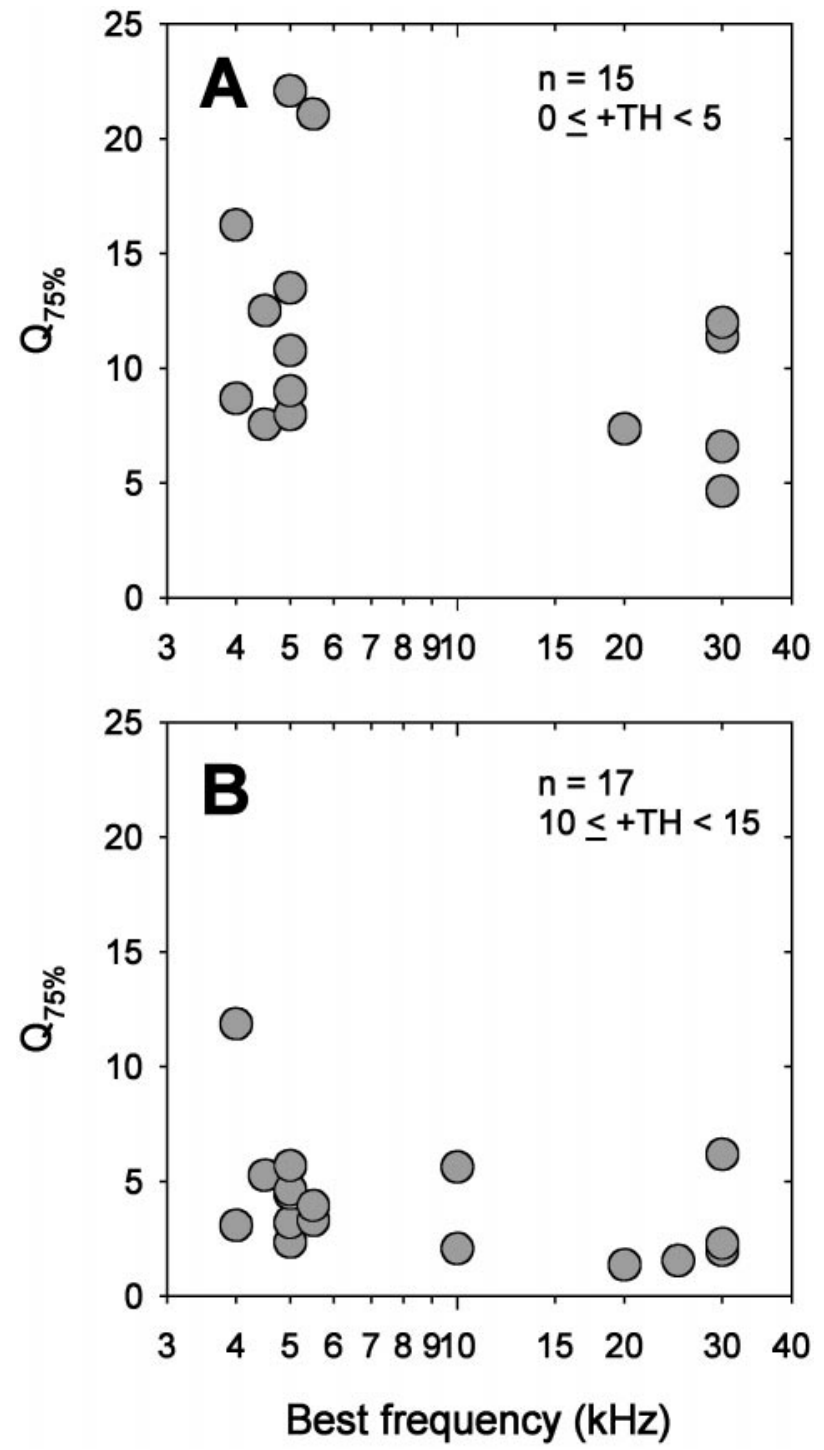

Figure 10. Frequency selectivity of ARFs. Frequency selectivity $\left(Q_{75 \%}\right.$ value) was calculated as BF divided by $75 \%$ bandwidth. Sample sizes and stimulus intensity above threshold $(+T H)$ are given at the top right. $A$, $Q_{75 \%}$ values from threshold to $<5 \mathrm{~dB}$ above threshold are plotted as a function of BF. Although there appears to be a trend toward sharper tuning for low-frequency ARFs, the difference between $Q_{75 \%}$ values of low-frequency and ultrasound ARF populations is not significant ( $t$ test, $d f=13 ; t=1.75 ; p=0.104) . B, Q_{75 \%}$ values from 10 to $<15 \mathrm{~dB}$ above threshold are shown. $Q_{75 \%}$ values are similar for the three ARF populations (ANOVA, $d f_{\text {effect }}=2 ; d f_{\text {error }}=14 ; F=1.18 ; p=0.335$ ).

tion from echolocating bats (Moiseff et al., 1978; for review, see Hoy, 1994). The behavioral roles of mid-frequency ARFs are less apparent. One possibility is that, like low-frequency ARFs, they mediate responses to communication signals. The songs of $T$. oceanicus do contain energy at higher harmonics of the dominant frequency. Behavioral experiments have shown that these may contribute to both the recognition and localization of the signals (Latimer and Lewis, 1986). A second possibility is that midfrequency ARFs, in particular those with high-pass characteristics, play a role in startle or avoidance responses.

Of particular interest is our finding that ARFs with CFs in the ultrasound range often have additional sensitivity peaks in the range of $4.5-5.5 \mathrm{kHz}$ (Fig. 7). Startle or avoidance responses,
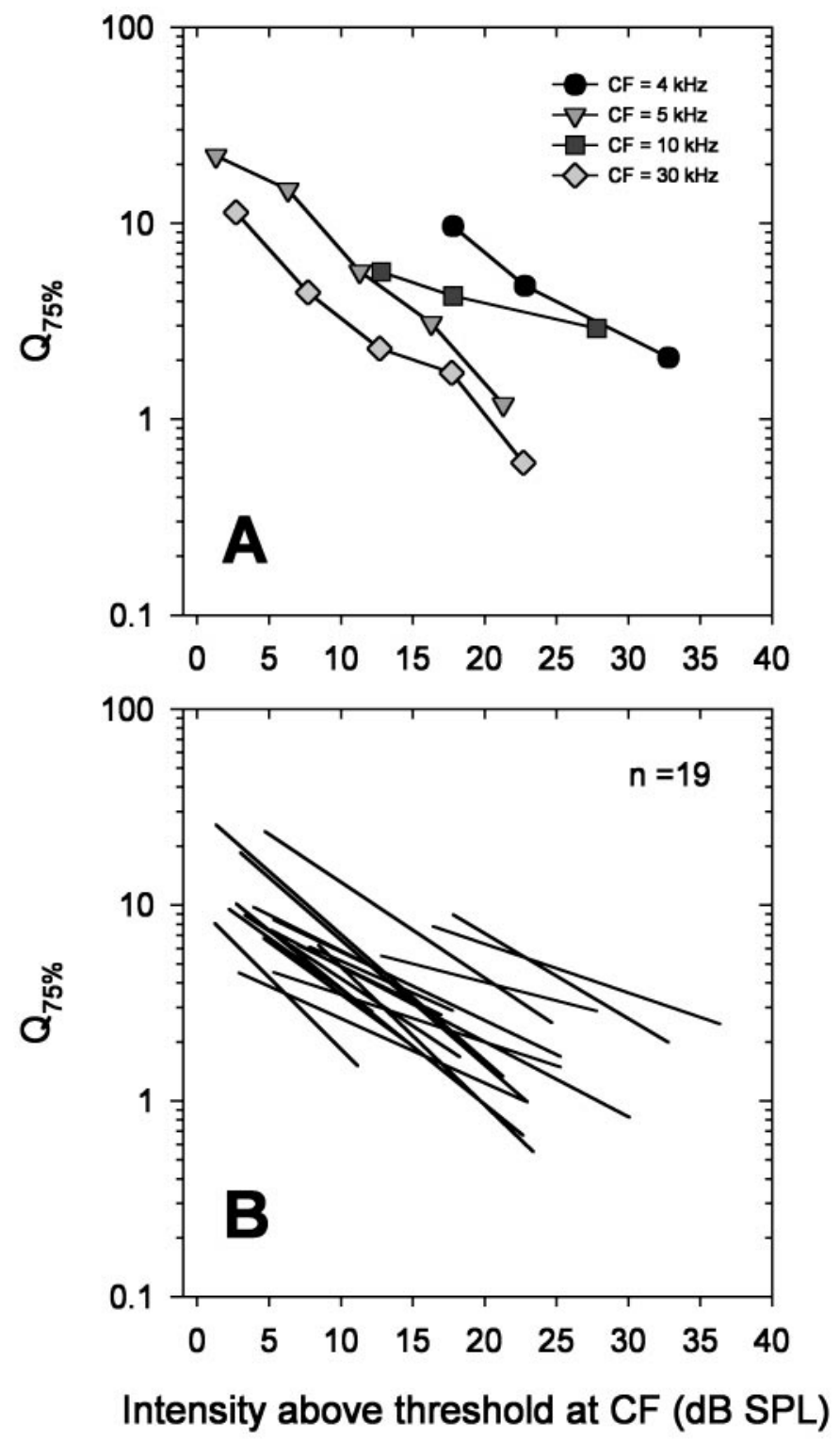

Figure 11. Frequency selectivity decreases with increasing intensity. $A$, The $Q_{75 \%}$ values of four representative ARFs are plotted as a function of increasing intensity above threshold at $\mathrm{CF}$ with different symbols and shades of gray. $B$, The changes of $Q_{75 \%}$ values with increasing intensity for a larger number of ARFs $(n=19)$ are plotted as slopes of regression lines of $\log Q_{75 \%}$ versus intensity. Regression lines were calculated from three to five values for each ARF.

similar to those evoked by ultrasound, are also occasionally elicited by low-frequency stimuli of sufficient intensity (Popov and Shuvalov, 1977; Nolen and Hoy, 1986). Our results raise the possibility that avoidance responses to low-frequency sounds occur because these stimuli activate ultrasound ARFs via their additional sensitivity peaks.

Behavioral experiments show that, although crickets detect sounds over a broad range of frequencies, they categorize their auditory world into only two frequency bands: a low-frequency band extending up to $\sim 15 \mathrm{kHz}$ and a high-frequency band extending from $15 \mathrm{kHz}$ into the ultrasound range (Wyttenbach et al., 1996). The existence of multiple sensitivity peaks implies that none of the three ARF populations will be activated exclusively by stimuli in their "own" frequency band. Particularly at higher 
intensities, identification of a sound as belonging to the "lowfrequency" or "high-frequency" categories may be ambiguous at the level of single receptor neurons. The sharp categorization of sound frequencies likely comes about as a result of interactions among interneurons.

\section{REFERENCES}

Balakrishnan R, Pollack GS (1996) Recognition of courtship song in the field cricket, Teleogryllus oceanicus. Anim Behav 51:353-366.

Capranica RR (1992) The untuning of the tuning curve: is it time? Semin Neurosci 4:401-408.

Eatock RA, Manley GA, Pawson L (1981) Auditory nerve fiber activity in the Tokay gecko. I. Implications for cochlear processing. J Comp Physiol [A] 142:203-218.

Eibl E, Huber F (1979) Central projections of tibial sensory fibers within the three thoracic ganglia of crickets (Gryllus campestris L., Gryllus bimaculatus DeGeer). Zoomorphologie 92:1-17.

Esch H, Huber F, Wohlers DW (1980) Primary auditory neurons in crickets: physiology and central projections. J Comp Physiol [A] 137:27-38.

Feng AS, Narins PM, Capranica RR (1975) Three populations of primary auditory fibers in the bullfrog (Rana catesbeiana): their peripheral origins and frequency sensitivities. J Comp Physiol 100:221-229.

Hoy RR (1994) Ultrasound acoustic startle in flying insects: some neuroethological and comparative aspects. In: Neural basis of behavioral adaptations (Schildberger K, Elsner N, eds), pp 227-241. Stuttgart, Germany: Gustav Fischer.

Hoy RR, Pollack GS, Moiseff A (1982) Species recognition in the field cricket, Teleogryllus oceanicus: behavioral and neural mechanisms. Am Zool 22:597-607.

Hutchings M, Lewis B (1981) Response properties of primary auditory fibers in the cricket Teleogryllus oceanicus (Le Guillou). J Comp Physiol [A] 143:129-134.

Imaizumi K, Pollack GS (1996) Anatomy and physiology of auditory receptors in the Australian field cricket Teleogryllus oceanicus. Soc Neurosci Abstr 22:1082.

Imaizumi K, Pollack GS (1997) Physiological properties of auditory receptors in the Australian field cricket Teleogryllus oceanicus. Soc Neurosci Abstr 23:1571.

Imaizumi K, Pollack GS (1998) Functional organization of axon terminals of auditory receptor fibers in Australian field cricket Teleogryllus oceanicus. 5th International Congress of Neuroethology 50.

Inglis M, Oldfield BP (1988) Tonotopic organisation of the auditory organ of the locust Valanga irregularis (Walker). J Comp Physiol [A] 164:49-53.

Johnstone BM, Saunders JC, Johnstone JR (1970) Tympanic membrane response in the cricket. Nature 227:625-626.

Kalmring K, Jatho M (1994) The effect of blocking inputs of the acoustic trachea on the frequency tuning of primary auditory receptors in two species of tettigoniids. J Exp Zool 270:360-371.

Kalmring K, Lewis B, Eichendorf A (1978) The physiological characteristics of the primary sensory neurons of the complex tibial organ of Decticus verrucivorus L. (Orthoptera, Tettigonioidae). J Comp Physiol [A] 127:109-121.

Kiang NY-S (1965) Discharge patterns of single fibers in the cat's auditory nerve. Cambridge, MA: MIT.

Köppl C (1997) Frequency tuning and spontaneous activity in the auditory nerve and cochlear nucleus magnocellularis of the barn owl Tyto alba. J Neurophysiol 77:364-377.

Larsen ON (1987) The cricket's anterior tympanum revisited. Naturwissenschaften 74:S92.

Latimer W, Lewis DB (1986) Song harmonic content as a parameter determining acoustic orientation behavior in the cricket Teleogryllus oceanicus (Le Guillou). J Comp Physiol [A] 158:583-591.

Liberman MC (1978) Auditory-nerve response from cats raised in a low-noise chamber. J Acoust Soc Am 63:442-455.

Libersat F, Murray JA, Hoy RR (1994) Frequency as a releaser in the courtship song of two crickets, Gryllus bimaculatus (de Geer) and Teleogryllus oceanicus: a neuroethological analysis. J Comp Physiol [A] 174:485-494.

Michelsen A (1971a) The physiology of the locust ear. I. Frequency sensitivity of single cells in the isolated ear. Z Vgl Physiol 71:49-62.
Michelsen A (1971b) The physiology of the locust ear. II. Frequency discrimination based upon resonances in the tympanum. Z Vgl Physiol 71:63-101.

Moiseff A, Pollack GS, Hoy RR (1978) Steering responses of flying crickets to sound and ultrasound: mate attraction and predator avoidance. Proc Natl Acad Sci USA 75:4052-4056.

Møller AR (1977) Frequency selectivity of single auditory-nerve fibers in response to broadband noise stimuli. J Acoust Soc Am 62:135-142.

Narins PM, Capranica RR (1976) Sexual differences in the auditory system of the tree frog Eleutherodactylus coqui. Science 192:378-380.

Nolen TG, Hoy RR (1986) Phonotaxis in flying crickets. I. Attraction to the calling song and avoidance of bat-like ultrasound are discrete behaviors. J Comp Physiol [A] 159:423-439.

Oldfield BP (1985) The tuning of auditory receptors in bushcrickets Hear Res 17:27-35.

Oldfield BP, Kleindienst HU, Huber F (1986) Physiology and tonotopic organization of auditory receptors in the cricket Gryllus bimaculatus DeGeer. J Comp Physiol [A] 159:457-464.

Paton JA, Capranica RR, Dragsten PR, Webb WW (1977) Physica basis for auditory frequency analysis in field crickets (Gryllidae). J Comp Physiol [A] 119:221-240.

Pollack GS (1994) Synaptic inputs to the omega neuron of the cricket Teleogryllus oceanicus: differences in EPSP waveforms evoked by low and high sound frequencies. J Comp Physiol [A] 174:83-89.

Pollack GS (1997) SWEEPS: a program for the acquisition and analysis of neurophysiological data. Comput Methods Programs Biomed 53:163-173.

Pollack GS, Faulkes Z (1998) Representation of behaviorally relevant sound frequencies by auditory receptors in the cricket Teleogryllus oceanicus. J Exp Biol 201:155-163.

Pollack GS, Huber F, Weber T (1984) Frequency and temporal patterndependent phonotaxis of crickets (Teleogryllus oceanicus) during tethered flight and compensated walking. J Comp Physiol [A] 154:13-26.

Popov AV, Shuvalov VF (1977) Phonotactic behavior of crickets. J Comp Physiol [A] 119:111-126.

Römer H (1983) Tonotopic organization of the auditory neuropile in the bushcricket Tettigonia viridissima. Nature 306:60-62.

Sachs MB, Woolf NK, Sinnott JM (1980) Response properties of neurons in the avian auditory system: comparisons with mammalian homologues and consideration of the neural encoding of complex stimuli. In: Comparative studies of hearing in vertebrates (Popper AN, Fay RR, eds), pp 323-353. New York: Springer.

Salvi RJ, Saunders SS, Powers NL, Boettcher FA (1992) Discharge patterns of cochlear ganglion neurons in the chicken. J Comp Physiol [A] 170:227-241.

Sams-Dodd F, Capranica RR (1994) Representation of acoustic signals in the eighth nerve of the Tokay gecko. I. Pure tones. Hear Res 76:16-30

Simmons AM, Buxbaum RC (1996) Neural codes for "pitch" processing in a unique vertebrate auditory system. In: Neuroethological studies of cognitive and perceptual processes (Moss CF, Shettleworth SJ, eds), pp 185-228. Boulder, CO: Westview.

Strausfeld NJ, Seyan HS, Wohlers D, Bacon JP (1983) Lucifer yellow histology. In: Functional neuroanatomy (Strausfeld NJ, ed), pp 132155. Berlin: Springer.

Stumpner A (1996) Tonotopic organization of the hearing organ in a bushcricket: physiological characterization and complete staining of auditory receptor cells. Naturwissenschaften 83:81-84.

Suga N (1990) Biosonar and neural computation in bats. Sci Am 262:60-68.

Weiss TF, Mulroy MJ, Turner RG, Pike CL (1976) Tuning of single fibers in the cochlear nerve of the alligator lizard: relation to receptor morphology. Brain Res 115:71-90.

Wyttenbach RA, May ML, Hoy RR (1996) Categorical perception of sound frequency by crickets. Science 273:1542-1544.

Yates, GK (1990) Basilar membrane nonlinearity and its influence on auditory nerve rate-intensity functions. Hear Res 50:145-162.

Young D, Ball E (1974) Structure and development of the auditory system in the prothoracic leg of the cricket Teleogryllus commodus (Walker). I. Adult structure. Z Zellforsch Mikrosk Anat 147:293-312.

Zaretsky MD, Eibl E (1978) Carrier frequency-sensitive primary auditory neurons in crickets and their anatomical projection to the central nervous system. J Insect Physiol 24:87-95. 